\title{
Cardiac murmurs auscultated in infancy and their importance to medical students
}

\begin{abstract}
The cardiac system is a substantial section on medical board examinations. Different levels of providers are expected to have a certain degree of knowledge of the cardiac system within their scope of practice. This article reviews basic infant heart murmurs, diastole vs. systolic classification, and history taking with physical examination components to obtain a differential diagnosis at the level of a third or fourth year medical student. Although benign heart murmurs are common in the pediatric population, it is critical to obtain a detailed history and be able perform a thorough physical examination in the infancy population during the critical postpartum period as some heart murmurs are life-threatening. The medical student should attempt to differentiate between the nature of the murmur-either benign or pathological-and resolve it in a reasonable time frame to ensure the health of the infant. Many pediatricians will automatically refer to a pediatric cardiologist for a specialist assessment; however, this can lead to an overflow of patients for the pediatric cardiologist, and influence patient turnaround and the ability to accept new patients.

This article outlines basic fetal circulation. It describes a focused cardiac physical examination for the scope of medical students in either third or fourth year (clinical rotations), and helps differentiate the heart murmur classifications. The information provided is a good starting point for neonatal/pediatric rotations and will prepare medical students for their end of rotation examination. It can also assist in pediatric cardiology elective rotation. The ability to classify each heart murmur based on physical exam findings is sometimes difficult for medical students transitioning to clinical rotations. The goal herein is to provide adequate and up-to-date information for the level of the audience.
\end{abstract}

Keywords: cardiac murmur, congenital heart disease, infant, medical students
Volume 7 Issue I - 2017

\author{
Theresa Rashdan, ${ }^{2}$ Ada Huang, ${ }^{2}$ Nicholas A \\ Kerna,' Tony Brown ${ }^{3}$ \\ 'College of Medicine, University of Science, Art and Technology, \\ BWI \\ ${ }^{2}$ Major General Hugh G Robinson Center for Medical Studies, \\ USA \\ ${ }^{3}$ Harvard University, USA
}

Correspondence: Nicholas A Kerna, College of Medicine, University of Science, Art and Technology, BWI, USA, Email nicholas.kerna@usat.edu

Received: May 22, 2017| Published: July 25, 2017
Abbreviations: ASD, atrial septal defect; G, gravida; PDA, patent ductus arteriosus; $\mathrm{P}$, para

\section{Introduction}

With the advent of technological advancement and diagnostic modalities in health care, simulation-based scenarios with decreasing emphasis on auscultation skills have reduced the medical student's abilities to auscultate, diagnose, and interpret heart sounds and murmurs. This leads to a lack of skilled primary care providers in the community and an increase in referrals to pediatric cardiologists. Although the majority of cardiac murmurs in infants are innocuous, it is imperative for medical students to understand cardiac murmurs, their physiology and their potential pathophysiology. Congenital heart disease affects 8/1000 live births. ${ }^{1}$ Congenital heart disease may be diagnosed prenatally, during birth or during hospitalization; however, many cases are asymptomatic for the first days and may go undetected. It is important to remember that most murmurs are not pathological, but a murmur may be the first or only clue to consequential congenital heart disease. ${ }^{2,3}$

\section{Case scenario}

You are completing your rotation in the newborn nursery making morning rounds. On your assessment of the impending discharge of a 36-hour-old female infant, you auscultate a murmur that was not present on your assessment of the previous day. The infant was born at 39.5 weeks via spontaneous vaginal delivery to a 26-year-old G1P0 mother who received limited prenatal care. The infant's APGAR score was 9 at one minute and 10 at five minutes. She weighed 2810 grams and measured $50 \mathrm{~cm}$ in length.

\section{Case dissection}

What are the important aspects that should be reviews? Focus on the maternal history. Important questions to ask include maternal age, serology testing, prenatal infections, past medical history and medications (prescribed and illicit). ${ }^{4}$ Consider maternal illnesses that may predispose the infant to congenital heart defects, such as systemic lupus erythematous, diabetes mellitus, rubella, Coxsackie viruses, herpes and Sjorgen's Disease. ${ }^{5}$. In addition, determine that the mother was not exposed to teratogens; such as alcohol, tobacco exposure, lithium, anticonvulsants or antidepressants. ${ }^{5}$ Finally, the medical student should inquire about family history to include congenital heart defects or genetic malformations. ${ }^{6}$

\section{Focused cardiology physical examination}

Heart sounds should be described by their intensity, location and radiation, quality, and timing in the cardiac cycle. ${ }^{3}$

Intensity: Heart sounds can be described as low-pitched or highpitched sounds. High-pitched sounds are often heard best with the diaphragm of the stethoscope. ${ }^{3}$

Location and Radiation: The location of the heart sound can help determine the etiology of the murmur. ${ }^{3}$ The standard listening areas on the chest (aortic, pulmonic, tricuspid and mitral) apply to both heart sounds and murmurs. ${ }^{3}$ It is also important to assess where the murmur radiates to within the heart.

Quality: Quality of the murmur is often described as mechanical, harsh, soft, vibratory or blowing in nature. ${ }^{3}$ Timing: The timing of the heart sounds is described in relation to the cardiac cycle: early, mid, or late systole; or early, mid or late diastole. ${ }^{3}$ 
The First Heart Sound (S1): The first heart sounds (S1) represents closure of the atrioventricular (mitral and tricuspid) valves. ${ }^{1}$ The resulting sound is in response to the ventricular pressure exceeding the atrial pressure at the onset of systole. ${ }^{1}$ The closure of the mitral and tricuspid valve occurs almost simultaneously; thus, $\mathrm{S} 1$ is considered a single sound. ${ }^{1}$

The Second Heart Sound (S2): The second heart sounds (S2) represents closure of the aortic and pulmonic valves. ${ }^{1}$ Typically, this is a high-pitched sound. Normally, the second heart sound (S2) is split which is often best heard at the pulmonic listening region that is located in the left upper sternal border. ${ }^{1}$ The aortic valve typically closes prior to the pulmonic valve. ${ }^{1}$ Variation does occur during respiration. ${ }^{1}$ Venous return to the right ventricle increases during inspiration and may be more delayed. ${ }^{1}$ A normal variant of S2 may be auscultated during respiration as it is normal for the split of the second heart sound to widen during inspiration and narrow during expiration. ${ }^{1}$ The Third Heart Sound (S3): The third heart sound may be a normal finding in children. ${ }^{1}$ The $\mathrm{S} 3$ is heard best at the cardiac apex. ${ }^{1}$. The $\mathrm{S} 3$ is heard during early diastole and represents rapid to slow ventricular filling. ${ }^{1}$ The Fourth Heart Sound (S4): The fourth heart sound is an abnormal late diastolic component that is heard as a result of a forcible atrial contraction in the presence of decreased ventricular compliance. ${ }^{1}$

\section{Abnormalities associated with wide splitting of S2}

1. Atrial septal defects (ASD): right ventricle overload; with an ASD, the split of S2 may be fixed as there is negligible change during inspiration and expiration.

2. Pulmonary stenosis: right ventricular outflow obstruction.

3. Complete right bundle branch block: delayed right ventricular depolarization.

4. A left bundle branch block: causes a paradoxical split split S2. A paradoxical split S2 occurs when the splitting is heard during expiration and disappears during inspiration, the opposite of the physiological split. Other abnormalities associated with a paradoxical split are S2 severed aortic stenosis and hypertrophic obstructive cardiomyopathy.

\section{Abnormalities associated with single S2}

1. Pulmonary Atresia: the pulmonary valve is missing

2. Aortic Atresia: the aortic valve is missing.

3. Truncus Arteriosus: a rare type of heart disease that in which a single blood vessel comes out of the right and left ventricles.

\section{Heart murmurs classification}

Heart murmurs are sounds generated by turbulent blood flow in the heart and blood vessels within the heart. ${ }^{5}$. Heart murmurs may be systolic, diastolic or continuous. ${ }^{5}$

Systolic Murmur Grades

I/VI: barely audible;

II/VI: faint but easily audible;

III/VI: loud murmur but no palpable thrill;

IV/VI: loud murmur with a palpable thrill;

V/VI: very loud murmur heard with stethoscope lightly placed on chest;
VI/VI: very loud murmur that may be heard without a stethoscope; and

Holosystolic Murmur: These murmur start at the beginning of S1 and continue to $\mathrm{S} 2 .{ }^{5}$

\section{Diastolic murmurs classification}

Diastolic murmurs are usually abnormal in their presentation and may be heard in early, mid-diastole or late-diastole. ${ }^{5}$ Early diastole murmurs immediately follow S2 in the cardiac cycle. Mid-diastolic murmurs result due to an increase flow through the mitral or tricuspid valves. ${ }^{5}$. Late-diastolic murmurs are due to pathological narrowing of the atrioventricular valves. ${ }^{5} \mathrm{~A}$ common continuous murmur present during systole and diastole is a patent ductus arterosus (PDA). ${ }^{5}$

\section{Discussion}

The history and physical examination are fundamental to identifying and detecting cardiac conditions. The next step is to categorize the differences between what is considered within normal limits versus abnormal. The presence of the four different heart sounds can be a normal finding in infants. ${ }^{7}$ Being able to differentiate which of the four heart sounds one is auscultating is a technique developed over time and with continued training. There are abnormalities associated with each sound pattern.

The heart sound classification is a technique used among healthcare providers to communicate their findings in a universal manner. Once a heart murmur is diagnosed and is not within normal limits for the infant's stage of life, the next appropriate step is referral to a pediatric cardiology specialist. ${ }^{3}$ When a heart murmur is diagnosed and is within normal limits for the infant's stage of life, close follow-up with the pediatrician is an appropriate step depending on the condition and any red flags observed from the history and physical examination. In some cases, an electrocardiogram and echocardiogram will be recommended. ${ }^{8}$

\section{Conclusion}

Heart murmurs can be differentiated into functional and pathological congenital heart murmurs. The clinical finding can be a sign of a heart disease, a heart defect, or benign based on the child's age. ${ }^{9}$ A most important skill is to determine if the results of the cardiac physical examination are normal or abnormal. ${ }^{10}$ Preceptors do not expect the medical student to be highly skilled in pediatric cardiology; however, they do expect the medical student to be actively engaged in reading up on the material required for a quality heart exam of infants and in improving their skill and expertise. Knowing how to perform a proper physical examination and auscultation, having a basic knowledge of heart murmurs and the classification thereof, and being able to differentiate between normal and abnormal findings are keys for a medical student's success on clinical rotations.

\section{Acknowledgments}

None.

\section{Conflicts of interest}

The authors declare that the research was conducted in the absence of any commercial or financial relationships that could be construed as a potential conflict of interest. 


\section{References}

1. Mesropyan L, Sanil Y. Innocent Heart Murmurs from the Perspective of the Pediatrician. Pediatr Ann. 2016;45(8):e306-e309.

2. Kang G, Xiao J, Wang Y, et.al. Prevalence and clinical significance of cardiac murmurs in schoolchildren. Arch Dis Child. 2015;100(11):10281031 .

3. Chantepie A, Soulé N, Poinsot J, et al. Heart murmurs in asymptomatic children:when should you refer. Arch Pediatr. 2016;23(1):97-104.

4. Gharehbaghi A, Borga M, Sjoberg BJ, et al. A novel method of discrimination between innocent and pathological heart murmurs. Medical Engineering and Physics. 2015;37(7):674-682.

5. Begic Z, Dinarevic SM, Pesto S2, et al. Evaluation of Diagnostic Methods in the Differentiation of Heart Murmur in Children. ACTA Inform Med. 2016;24(2):94-98.

6. Sachkey AH. Prevalence and diagnostic accuracy of heart disease in children with asymptomtatic murmurs. Cardiol Young. 2016;26(3):446450 .

7. Kardasevic M, Kardasevic A. The importance of heart murmur in the neonatal period and justification of echocardiographic reivew. Med Arch. 2014;68(4):282-284

8. Chelo D, Nguefack F, Menanga AP, et al. Spectrum of heart disease in children:an echocardiographic study of 1,666 subjects in a pediatric hospital, Yaounde, Cameroon. Cardiovascular Design and Therapy. 2016;6(1):10-19.

9. Pedrosa J, Castro A, Vinhoza TT. Automatic heart sound segmentation and murmur detection in pediatric phonocardiograms. Conf Proc IEEE Eng Med Biol Soc. 2014;2014:2294-2297.

10. Khushu A, Kelsall AW, Usher-Smith JA. Outcome of Children with heart murmurs referred from general practice to a pediatrician with expertise in cardiology. Cardiology in the Young. 2015;25(1):123-127. 